\title{
Adolf Lorenz und Tomáš Garrigue Masaryk - eine außergewöhnliche Begegnung
}

\author{
Gerold Holzer (D) · Helena Kokešová
}

Eingegangen: 23. August 2019 / Angenommen: 22. Dezember 2019 / Online publiziert: 28. Januar 2020

(C) Der/die Autor(en) 2020

Zusammenfassung Adolf Lorenz, der Begründer der Orthopädie in Österreich und Vater des späteren Nobelpreisträgers Konrad Lorenz, und der Gründer und erste Präsident der tschechoslowakischen Republik, Tomáš G. Masaryk, standen in Kontakt miteinander. In den siebziger und achtziger Jahren des 19. Jahrhunderts studierten beide an der Universität Wien: der um vier Jahre ältere Masaryk Philosophie und Lorenz Medizin. Die ersten Treffen fanden im Seziersaal des Anatomischen Institutes statt. Ihnen beiden gelangen (wissenschaftliche) Karrieren und sie wurden prominente Persönlichkeiten. Fast 40 Jahre nach der ersten zufälligen Begegnung erinnerte sich Lorenz an seinen ehemaligen Schüler, inzwischen Präsident der tschechoslowakischen Republik, und nahm mit ihm Briefkontakt auf. Die im Masaryk-Institut in Prag aufbewahrte Korrespondenz soll hier vorgestellt werden.

Schlüsselwörter Österreichisch-Ungarische Monarchie · Medizinstudium · Korrespondenz • Memoiren · Politik

Die gesamte Korrespondenz findet sich im Masaryk-Institut und Archiv der Akademie der Wissenschaften der Tschechischen Republik (MUA), Abteilung Archiv des Instituts von T.G. Masaryk, Bestand T.G. Masaryk, Karton 700, Signatur KOR-II-7 und Karton 701, Signatur KOR-II-15.

Univ. Prof. Dr. med. G. Holzer ( $₫)$

Universitätsklinik für Orthopädie und Traumatologie, Allgemeines Krankenhaus, Medizinische Universität Wien, Währinger Gürtel 18-20, 1090 Wien, Österreich gerold.holzer@meduniwien.ac.at

Dr. phil. H. Kokešová

Masaryk-Institut und Archiv der Akademie der

Wissenschaften der Tschechischen Republik (Masarykův

ústav a Archiv Akademie věd České republiky),

Gabčíkova 10, 18200 Prag 8, Tschechien

\section{Adolf Lorenz und Tomáš Garrigue Masaryk-an extraordinary encounter}

Summary Adolf Lorenz, the founder of orthopedics in Austria and father of the later Nobel laureate Konrad Lorenz, and the founder and first president of the Czechoslovak Republic, Tomáš G. Masaryk, were in contact. In the seventies and eighties of the nineteenth century both studied at the University of Vienna: Masaryk, four years older, Philosophy and Lorenz Medicine. The first meetings took place in the dissecting room of the Anatomical Institute. Later both entered (scientific) careers and became wellknown personalities. Almost 40 years after the first coincidental encounter, Lorenz remembered his former student, meanwhile president of the Czechoslovak Republic, and made contact with him. The correspondence kept in the Masaryk Institute in Prague will be presented here.

Keywords Austro-Hungarian Monarchy · Study of medicine $\cdot$ Correspondence $\cdot$ Memoirs · Politics

\section{Adolf Lorenz und Tomáš Garrigue Masaryk Studienjahre}

Als eine der großen Metropolen Europas bildete Wien im ausgehenden 19. Jahrhundert („Fin-de-Siecle“) [1] das politische, gesellschaftliche, kulturelle und wissenschaftliche Zentrum des Vielvölkerstaates der Habsburgischen Donaumonarchie. In diesem Schmelztegel versammelte sich nicht nur die Elite des gesamten Reiches, sondern die Stadt zog auch Menschen aus aller Welt an und bildete so den Nährboden und das Ambiente für viele der kommenden Umwälzungen.

Zufällig trafen hier auch der in Schlesien geborene Adolf Lorenz und der aus Mähren stammende Tomáš 


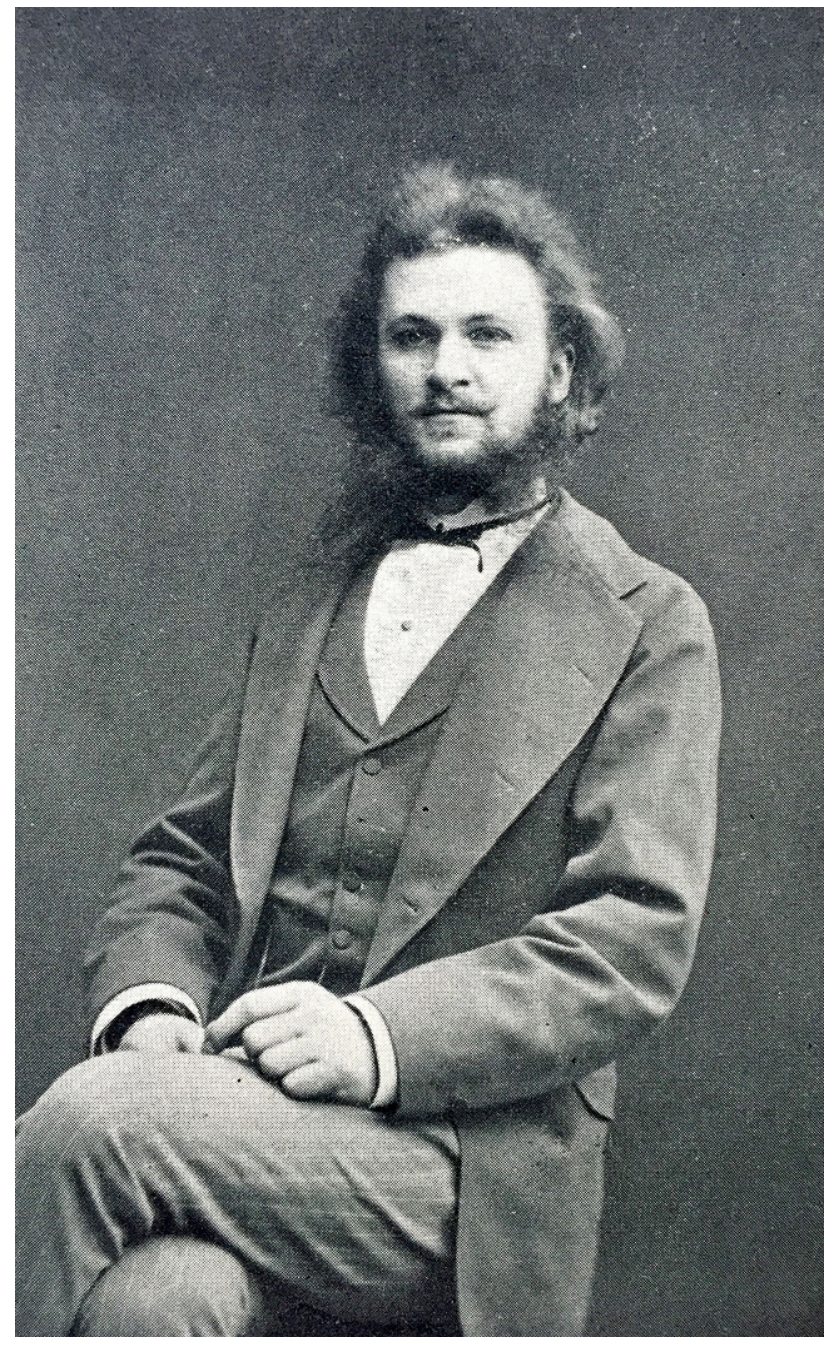

Abb. 1 Porträt von Adolf Lorenz als Medizinstudent. (Aus: [16])

Masaryk zusammen. Masaryk kam während der Gymnasialzeit nach Wien, studierte nach der Matura am Akademischen Gymnasium an der Universität Wien Philosophie, wurde 1876 promoviert und habilitierte 1879 [2].

Adolf Lorenz, 1854 in Weidenau im Vorland des Altvatergebirges (damals Oberschlesien) geboren ${ }^{1}$, besuchte das Gymnasium in St. Paul im Lavanttal und kam erst nach der Matura im Staats-Obergymnasium in Klagenfurt nach Wien, um hier das Medizinstudium zu beginnen [3, S. 126-130].

Trotz der unterschiedlichen Studienrichtungen kam es zu Beginn der 1880er Jahre zu Begegnungen der beiden, als Lorenz, damals noch Medizinstudent (Abb. 1), als Demonstrator im Anatomischen Institut

\footnotetext{
1 Die Stadt Weidenau befand sich im Herzogtum Schlesien (Oberschlesien). Das Herzogtum Ober- und Niederschlesien, inoffizielle Bezeichnung Österreichisch-Schlesien, war ein inkorporierter Teil der Länder der Böhmischen Krone und damit der Habsburgermonarchie. 1918 fiel ein Großteil Österreichisch-Schlesiens der neu gegründeten Tschechoslowakei zu.
}

von Professor Karl Langer in der Schwarzspanierstraße arbeitete. Ein ihm damals unbekannter Mann bestellte für sich und einen Freund ein Privatissimum. Da die beiden Interessenten keine Zulassung als Medizinstudenten hatten, musste der Kurs im Geheimen an „dunklen Winterabenden“ stattfinden. Lorenz wurde von zwei Männern aufgesucht, deren Namen er nicht genau verstand. ${ }^{2}$ Deshalb nannte er sie „den Dunklen“ und „den Lichten“3 [4, S. 1-3].

In der letzten Anatomiestunde nahm der „Dunkle“ unbemerkt ein Skalpell zur Hand und führte oberhalb des Handgelenkes einen schrägen Schnitt aus, der sich tief in die Weichteile bohrte. „Was habe ich nun angerichtet?“, fragte dieser den Lehrer. „Sie haben zwei wichtige Nerven, eine große Schlagader und mehrere Sehnen durchschnitten, [...] Für den Lebenden würde die kleine Wunde zum allermindesten die vollkommene Unbrauchbarkeit der Hand bedeuten, welche nach einiger Zeit die Form einer abschreckenden Kralle annehmen müßte“, antwortete Lorenz und fügte hinzu: „Sie sind ein schlechter Anatom, Herr Doktor! Ein Glück, dass Sie Philosoph sind.“ [4, S. 2]

Nach diesen eher zufälligen Treffen trennten sich die Wege der beiden wieder. Lorenz arbeitete nach der Promotion 1880 als Operateur, später als Assistent von Prof. Eduard Albert an der I. Chirurgischen Klinik. Da an Lorenz' Händen durch die Verwendung antiseptischer Desinfektionsmittel beim Operieren Karbolekzeme auftraten, wurde ihm die Ausübung der klassischen Chirurgie unmöglich gemacht. Albert verwies ihn auf das damals noch unbebaute Feld der Ortho-

2 Ein Jahr nach dem Erscheinen von Lorenz' Artikel in der „Neu-
en Freien Presse“ wurde in der „Tribuna“ in einer Ergänzung von
Jaromír Doležal die Identität des zweiten Mannes enthüllt: Es
handelte sich um Anton Oetzelt-Newin, einen Privatgelehrten,
der damals in der Nähe von Adolf Lorenz' Wohnung in Wien leb-
te. Anton Oetzelt-Newin (1854-1922) war auch Philosoph, dem
in Wien trotz erfolgreich beendetem Habilitationsverfahrens aus
formalen Gründen vom Ministerium die Venia legendi verwei-
gert wurde. Schließlich habilitierte er in Bern, wo er 1890 zu lesen
begann $[4,5]$.

${ }^{3}$ Im Artikel führte er an, dass der „Lichte“ gesagt habe: „Mein Name tut nichts zur Sache“; der „Dunkle stellte sich vor: ,Mein Name ist Dr ...', es folgten einige sonore Silben mit scharfem, hohem Schlußklang, welche der sich gleichzeitig vorstellende Student zu einem klaren Wortbild nicht bringen konnte“. In späteren Jahren veröffentlichte Lorenz diese Anekdote mit einigen Abweichungen. In einer Erinnerung vom September 1937 schrieb er beispielsweise, dass er bei der Vorstellung einen sonderbar fremd klingenden Namen in der Art von „Marrick“ oder „Arrick“ gehört und gar nicht versucht habe, sich diesen zu merken [7, 8]. Zum Jahresende 1937 sagte Lorenz, dass der hellhaarige Gast seinen Begleiter als „Doktor Aryk“ und sich selbst als „Doktor Zelt“ (oder mit ähnlichen Namen) vorgestellt habe [9]. Ähnlich auch in seinem Erinnerungen: „Ich nannte meine beiden Schüler den ,Lichten ' und den ,Dunklen', da ich mir die Namen, besonders den des letzteren nicht merken konnte. In seinem Munde klang der Name wie das Schlagen eines Hammers gegen den eisernen Amboß. Ich vernahm Laute wie: schick - schrak - schrik“ [10, S. 101-102]. 


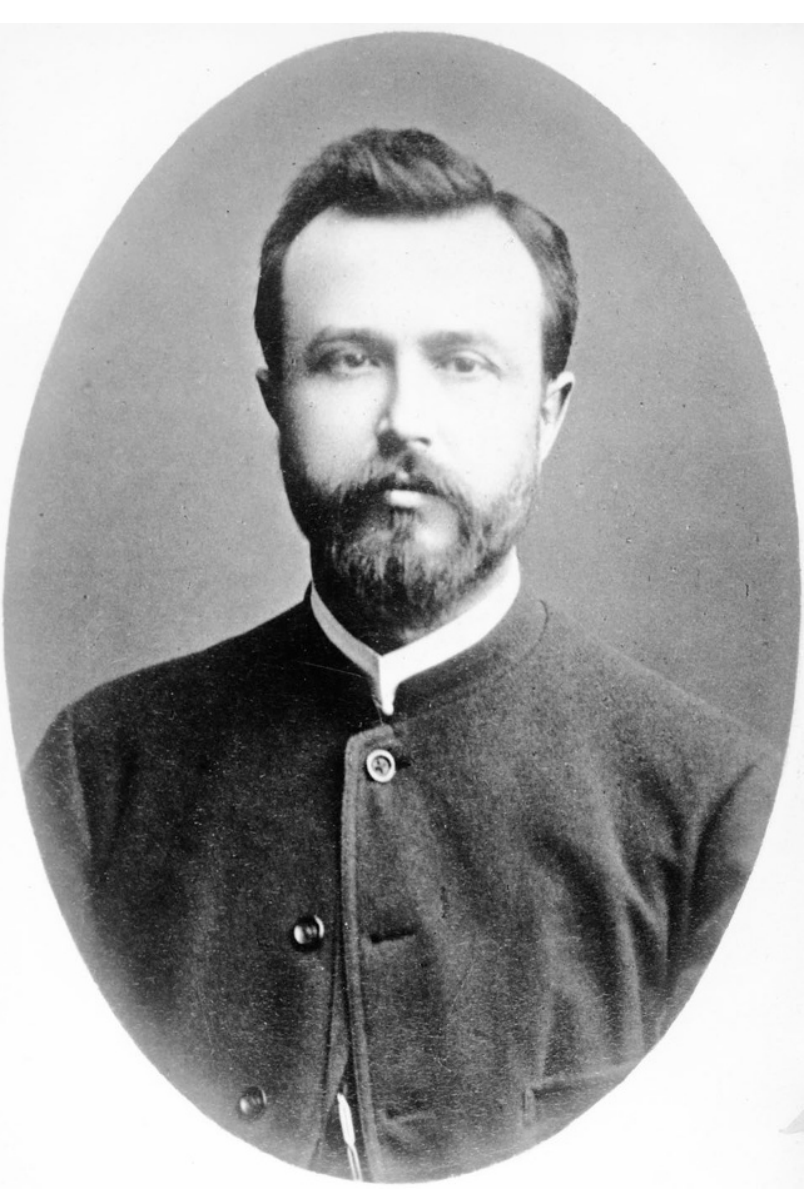

Abb. 2 Porträt von T.G. Masaryk aus Jahr 1882 nach der Ankunft in Prag. (MUA, Bestand Institut von T.G. Masaryk, Signatur 320)

pädie, in dem sich Lorenz profilieren konnte. Lorenz wurde 1884 habilitiert und später Professor für Orthopädie in Wien. Vor allem durch „seine unblutige Operation“ (Reposition und Fixation) bei kongenitalen Hüftgelenksluxationen gelangte er zu Weltruhm [6]. Höhepunkt seiner Karriere war 1902 in Chicago die Therapie von Lolita Armour, der Tochter des Ehepaares J. Ogden Armour, damals eine der reichsten Familien der Welt.

Masaryk übersiedelte 1882 als außerordentlicher Professor für Philosophie an die Tschechische Universität in Prag (Abb. 2). 1891 wurde er in den Reichsrat und den böhmischen Landtag gewählt, legte aber 1893 sein Mandat nieder. 1907 kehrte er in das Abgeordnetenhaus zurück. Ab 1897 widmete Masaryk sich als ordentlicher Professor neben seiner Lehrtätigkeit der staatspolitischen Erziehung der Tschechen, für die er eine nationale Wiedergeburt anstrebte. Auch sonst ergriff er vielfach in Fragen des öffentlichen Lebens (Ritualmordprozeß von Polna, Wahrmund-Affäre) und der Außenpolitik (Agramer Prozeß, Friedjung-Prozeß) das Wort.

Nach Ausbruch des Ersten Weltkrieges stellte sich Masaryk ganz auf eine westliche Orientierung ein und ging deshalb in die Emigration nach England und in

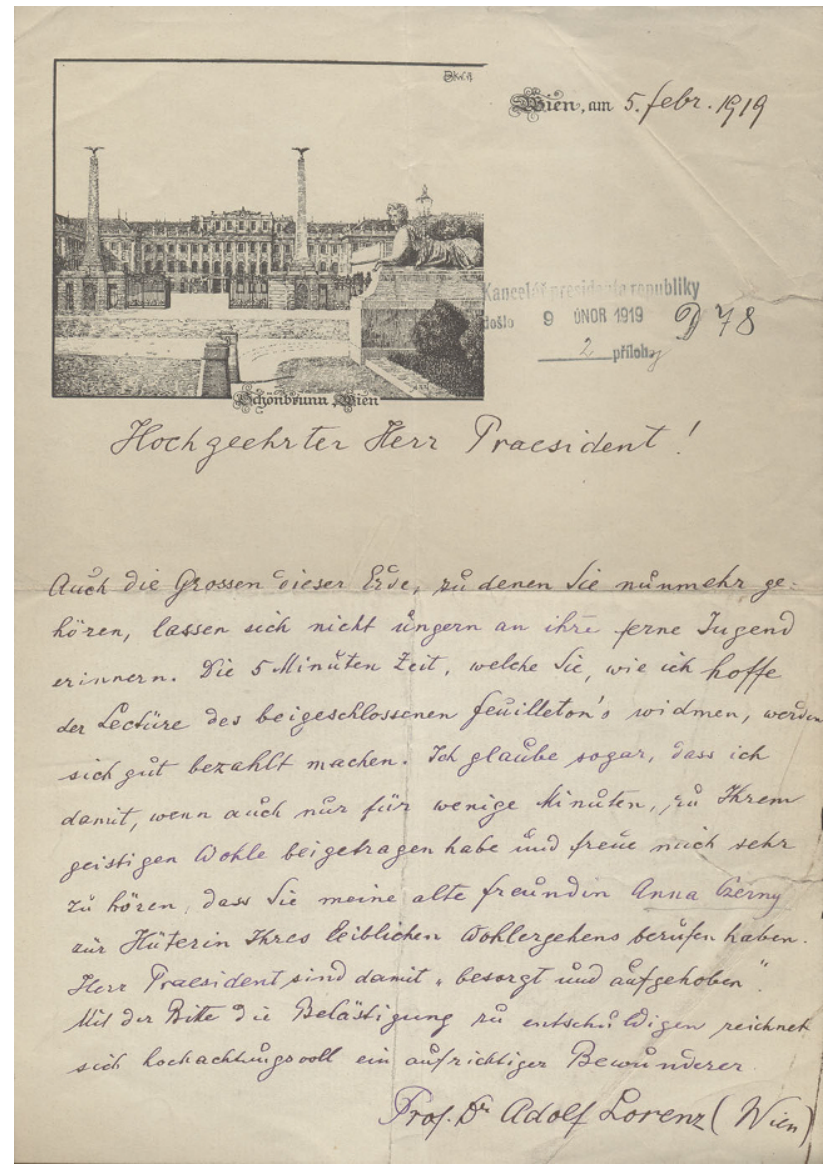

Abb. 3 A. Lorenz an T.G. Masaryk 05.02.1919. (MUA, Bestand T.G. Masaryk, Karton 700, Signatur KOR-II-7)

die USA. Dort arbeitete er an der Vorbereitung einer nationalen Revolution der Tschechen, die im Rahmen einer demokratischen Neugestaltung Europas erfolgen sollte. Dabei gelang es ihm, die Slowaken für den Gedanken eines gemeinsamen tschechoslowakischen Staates zu gewinnen, der aus den Trümmern der österreichisch-ungarischen Donaumonarchie aufgebaut werden sollte. Durch Einwirkung auf Präsident Wilson erreichte Masaryk 1918 die Anerkennung einer tschechoslowakischen Exilregierung, an deren Spitze er trat. Nach Kriegsende kehrte er als „Befreier-Präsident" nach Prag zurück, wo er 1918 zunächst in $a b$ sentio zum Staatsoberhaupt gewählt wurde. ${ }^{4}$

\section{Fast vierzig Jahren später}

Fast vierzig Jahre waren vergangen, bevor Lorenz in jenem Doktor der Philosophie und ehemaligen Anatomieschüler, den Präsidenten der Tschechoslowakischen Republik erkannte und beschloss, seine Begeg-

\footnotetext{
${ }^{4} 1878$ heiratete Masaryk die Amerikanerin Charlotte Garrigue und fügte ihren Namen seinem Namen hinzu. Mehr zu Masaryk http://www.biographien.ac.at/oebl?frames=yes und https://de. wikipedia.org/wiki/Tom\%C3\%A1\%C5\%A1_Garrigue_Masaryk.
} 


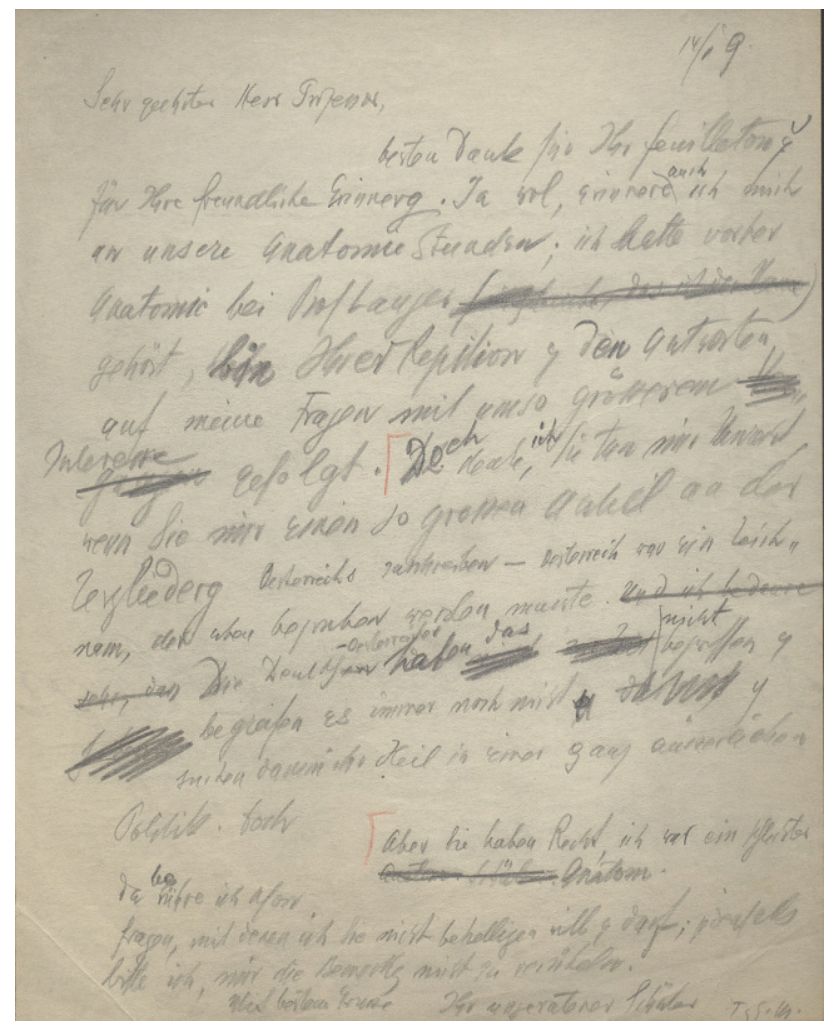

Abb. 4 T.G. Masaryk an A. Lorenz 14.02.1919, Brief-Konzept. (MUA, Bestand T.G. Masaryk, Karton 700, Signatur KORII-7)

nung mit Masaryk auf der Anatomie in Wien in einem Feuilleton, das am 4. Februar 1919 in der Wiener „Neuen Freien Presse“ erschien, zu veröffentlichen. Er schloss mit dem Satz:

Herr Präsident der czechoslowakischen Repu-

blik, Thomas G. Masaryk - ihr seid ein großer

Mann geworden, aber ein schlechter Anatom geblieben! [4, S. 3]

Am folgenden Tag schrieb Lorenz einen Brief an Masaryk (Abb. 3) und legte den Zeitungsartikel Der schlechte Anatom bei. Lorenz bat Masaryk dem beigelegten Feuilleton wenigstens fünf Minuten seiner Zeit $\mathrm{zu}$ widmen. Nebenbei erwähnte er, dass er froh sei, dass Masaryk die gemeinsame Bekannte, Anna Černá, in seine Dienste übernommen habe („Herr Präsident sind damit ,besorgt und aufgehoben“") [11]. Lorenz' Artikel wurde umgehend ins Tschechische übersetzt und bereits am 6. Februar 1919 in der „Tribuna“ unter dem Titel Špatný anatom publiziert [12].

Der Stempel der Präsidialkanzlei verrät, dass Lorenz' Brief mit den Anlagen am 9. Februar 1919 in Prag eingegangen war [11]. Masaryks Antwort ließ nicht lange auf sich warten. Das Original von Masaryks Antwort an Lorenz liegt nicht vor, aber im Masaryk-Bestand ist das Konzept seines Antwortschreibens vom 14. Februar 1919 überliefert (Abb. 4). Darin reagier-

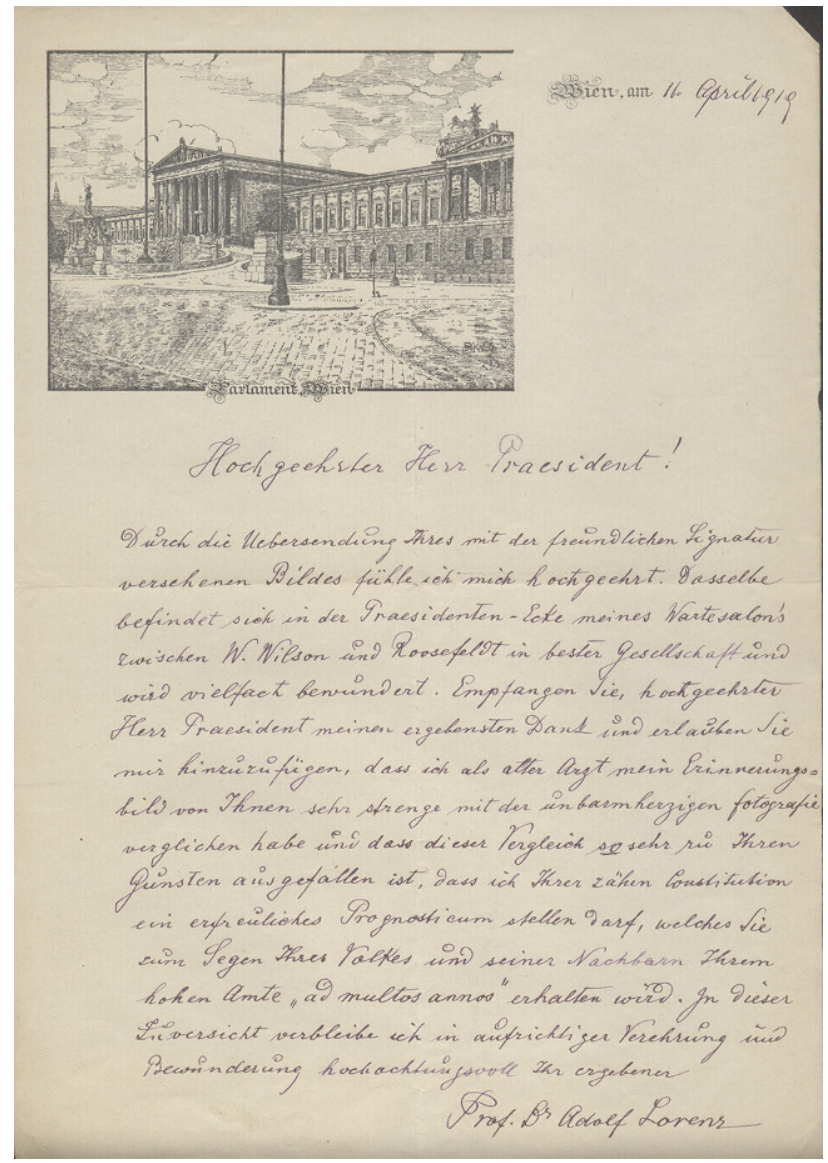

Abb. 5 A. Lorenz an T.G. Masaryk 11.04.1919. (MUA, Bestand T.G. Masaryk, Karton 700, Signatur KOR-II-7)

te Masaryk nicht nur auf Lorenz' Feuilleton, sondern auch auf den Begleitbrief. ${ }^{5}$

Masaryk bedankte sich für den Feuilletonartikel. Er selbst erinnerte sich ebenfalls an die Anatomiestunden, in denen er Lorenz' Repetition und die Antworten auf seine Fragen mit großem Interesse verfolgt hatte. Er stimmte Lorenz' Feststellung zu, dass er ein schlechter Anatom gewesen sei. Dann fuhr Masaryk allerdings fort:

Doch denke ich, Sie tun mir Unrecht, wenn Sie mir einen so grossen Anteil an der Zerglieder[un]g Oesterreichs zuschreiben - Oesterreich war ein Leichnam, der aber begraben werden musste. Die Deutsch-Oesterreicher haben das nicht begriffen und begreifen es immer noch nicht und suchen darum ihr Heil in einer ganz äusserlichen Politik. [11]

\footnotetext{
${ }^{5}$ In Tschechien ist nur die tschechische Übersetzung des Masaryk-Briefs bekannt, die in den zitierten Artikeln $[5,9]$ veröffentlicht wurde; letztens in dem Artikel von Ludmila Hlaváčková [13]. Der gesamten Briefwechsel Lorenz - Masaryk wurde im Herbst 2019 in der Zeitschrift Práce $\mathrm{z}$ dějin Akademie věd [Studies in the History of the Academy of Sciences] veröffentlicht [14].
} 


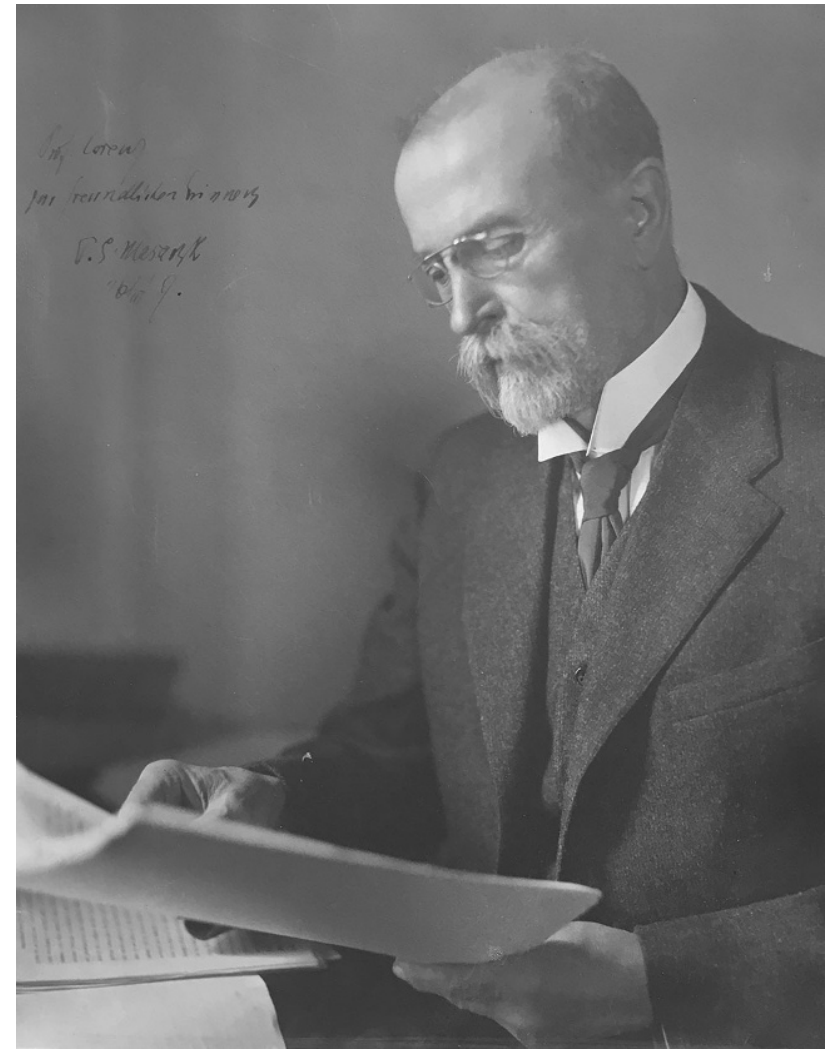

Abb. 6 Porträt von T.G. Masaryk aus dem Jahr 1919 mit der Widmung für Lorenz. (Foto: G. Holzer)

Den Brief, der mit einem Gruß des „ungeratene[n] Schüler[s]“ endete, schloss er mit der Bitte, dass Lorenz ihm diese politische Anmerkung nicht übelnehmen solle.

Lorenz unterhielt mit Masaryk bis zu dessen Lebensende gelegentliche freundschaftliche Briefkontakte. Als Beweis mag Lorenz' Brief vom 11. April 1919 dienen (Abb. 5), in dem er Masaryk für die zugesandte Fotografie mit Widmung (Abb. 6) dankte. Lorenz stellte dieses Bild zu den bereits vorhandenen Fotografien der amerikanischen Präsidenten Woodrow Wilson und Theodore Roosevelt in die Präsidentenecke im Warteraum seiner Ordination in der Rathausstraße 21 [11]. Dort können sie auch heute noch besichtigt werden (Abb. 7). ${ }^{6}$

Im Masaryk-Institut befindet sich ein weiterer Brief Lorenz' vom 20. Januar 1920 (Abb. 8). Offensichtlich reagierte Lorenz darin auf Masaryks Neujahrswünsche. Er erwidert diese und wünschte Masaryk zu dessen nahendem 70. Geburtstag (7. März 1920), die Gesundheit noch lange genießen zu können. Außerdem erwähnt er seinen Assistenten Dr. Saxl [11]. Der Brief dürfte mit einem Geschehen zusammenhängen, über das Lorenz in seinem Brief von Dezember

6 Adolf und Albert Lorenz Gedenkstätte, Rathausstraße 21, Wien. http://www.medacad.org/adolf-lorenz/index.html

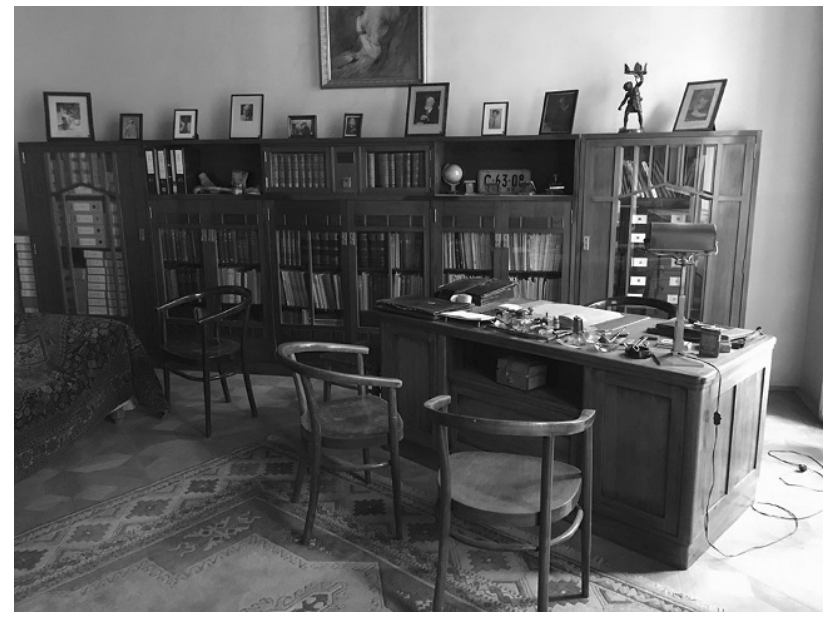

Abb. 7 Lorenz' Arbeitszimmer mit Präsidentenporträts, zweites von rechts T.G. Masaryk. (Foto: G. Holzer)

1937 berichtete. Lorenz hatte sich mit einer Bitte um Hilfe für einen ehemaligen Schüler an Masaryk gewandt, bekam allerdings von Masaryk eine Abfuhr [9, S. 1720].

Im gleichen Artikel findet sich auch eine historisch interessante Anmerkung von Lorenz über seinen eigenen Lehrer, den berühmten Chirurgen Eduard Albert. Lorenz beschrieb ihn als tschechischen Patrioten mit deutscher Bildung, der sich in Wien in Wort und Schrift um die deutsch-tschechische Versöhnung bemüht habe. Eines Tages sei Albert sehr aufregt und mit strahlendem Gesicht zu Lorenz gekommen und habe diesen gebeten, ihn anzusehen: Seine Frage war, ob er in seinem Gesicht nicht den Abglanz der kaiserlichen Gunst und Gnade erkennen könne. Albert war nämlich gerade von Seiner Majestät, dem Kaiser, wegen seiner Versuche um eine Versöhnung der Völker Österreichs mit Lob überschüttet worden [9, S. 1720]. Vermutlich stammt diese Anekdote von Alberts Audienz bei Kaiser Franz Joseph im Jahr 1895. ${ }^{7}$

Lorenz' Erinnerungen (Abb. 9) zufolge, ruhten die (brieflichen) Beziehungen zu Präsident Masaryk nach 1920 für längere Zeit, bis sie in den letzten fünf bis acht Lebensjahren Masaryks über Lorenz' alte Bekannte Anna Černá wieder neu geknüpft wurden. Anna Černá war nicht nur eine gute Bekannte von Lorenz, sie kannte auch Eduard Albert sehr gut, der mit ihrem Bruder Josef Černý das Gymnasium besucht hatte. Anna Černá war Verwalterin der Landesheilanstalt in Brünn, wo Alberts Freund Viktor Mucha den Direktorenposten innehatte. Von dort ging sie zusammen mit Direktor Mucha nach Wien an das Allgemeine Krankenhaus, wo sie in der Zeit der Habsburgermonarchie Oberverwalterin war [15].

Nach der Gründung der Tschechoslowakischen Republik verließ sie Wien und wurde mit der Führung

7 Unsere Annahme basiert auf einem Eintrag im Gedenkbuch der Albert-Villa in Senftenberg (Žamberk), das im dortigen Stadtmuseum (Městské muzeum v Žamberku) aufbewahrt wird. 


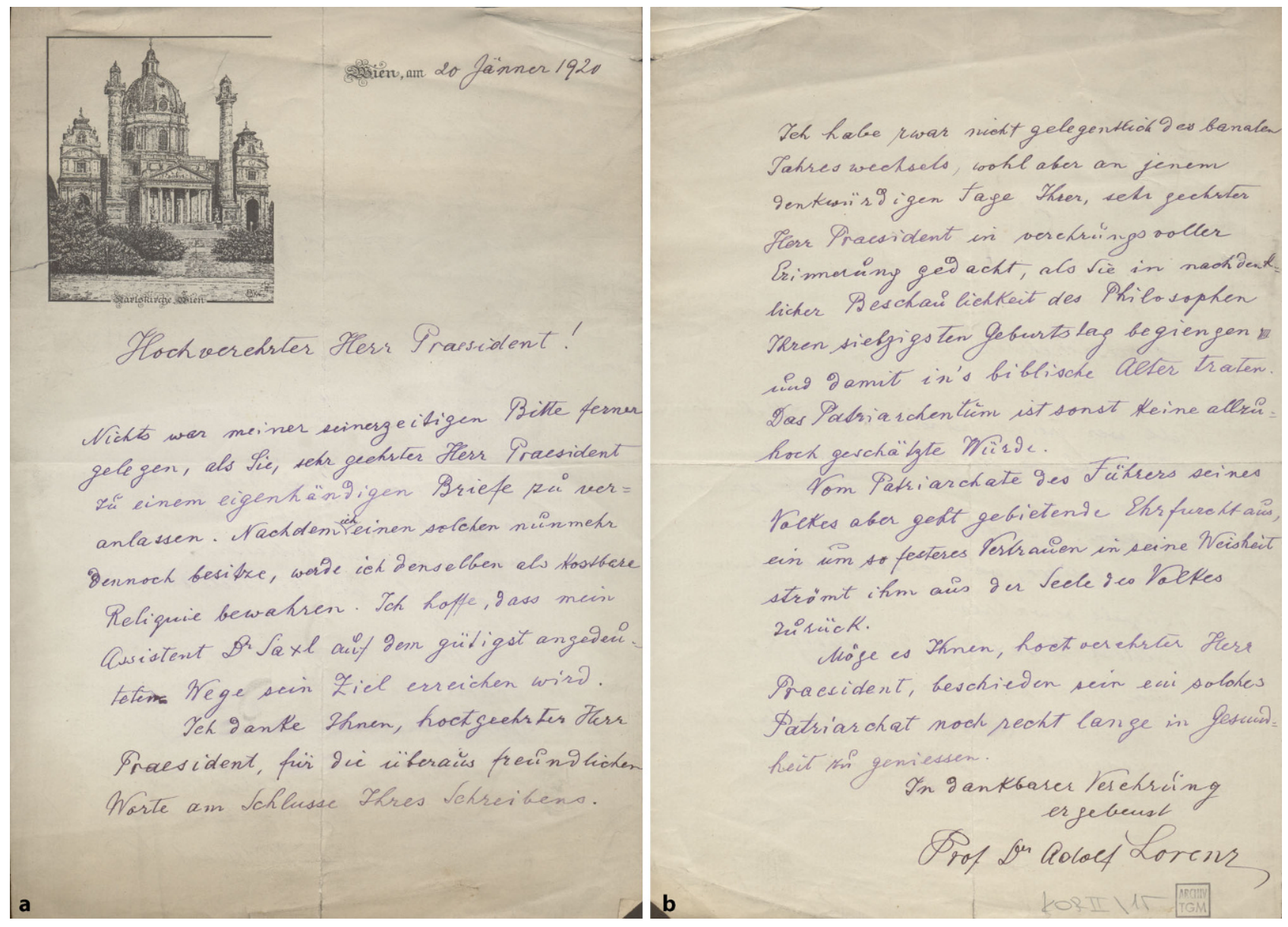

Abb. 8a,b A. Lorenz an T.G. Masaryk 20.01.1920. (MUA, Bestand T.G. Masaryk, Karton 701, Signatur KOR-II-15)

des Präsidentenhaushaltes auf Schloss Lana (Lány) betraut. In dieser neuen Position bezeichnete Lorenz sie scherzhaft als „Obersthofmeisterin Seiner Majestät des ungekrönten Königs der Tschechoslowakei“" [7, 9, S. 1720].

Lorenz erinnerte sich, dass Anna später einmal pro Jahr nach Wien kam, um ihre Schwester zu besuchen, und ihm dann jedes Mal Grüße des „Herrn Präsidenten" ausrichtete [7, 9, S. 1720]. Im Laufe der folgenden Jahre schickte Lorenz Masaryk weiterhin gelegentlich Artikel weniger mit politischem, eher mit allgemeinem Inhalt.

In einem Artikel hat Lorenz auch die Enthüllung einer Gedenktafel im heimatlichen Weidenau angesprochen: Als er in seiner Heimatstadt, die nach dem Ersten Weltkrieg an die Tschechoslowakei gefallen war, geehrt werden sollte, habe Präsident Masaryk die Kommunalpolitiker daran erinnert, dass er ein alter Freund von Lorenz sei und einst mit ihm zusammengearbeitet habe [7]. Der Präsident habe die Stadtväter in einem Erlass für die Absicht, ihn zu ehren, gelobt [9, S. 1720]. Detaillierter beschrieb Lorenz diese Ereignisse in seinen Memoiren [10, S. 395-403].

Masaryk wurde 1920, 1927 und 1934 zum Staatsoberhaupt wiedergewählt. 1935 legte er dieses Amt aus Altersgründen zurück. In seiner Person verkörperte sich die tschechoslowakische Staatsideologie der Zwischenkriegszeit.

Lorenz letzte Botschaft an Masaryk war die „Warnung, nicht hundert Jahre alt zu werden“. Den Artikel publizierte er im Neuen Wiener Tagblatt noch zu einer Zeit, als Masaryk sich guter Gesundheit erfreute. Der Autor konnte nicht ahnen, dass der „Philosoph von Lana“ sich seine Warnung so bald und so gründlich zu Herzen nehmen würde [7]. Der Artikel wurde unter dem Titel Mi̊j žák Masaryk ins Tschechische übersetzt und drei Tage nach dem Tod T.G. Masaryks am 14. September 1937 veröffentlicht [8].

Adolf Lorenz überlebte Masaryk um neun Jahre. Für seine Behandlungsmethode der angeborenen Hüftluxation wurde Lorenz für den Nobelpreis vorgeschlagen, letztlich fehlte dafür jedoch eine Stimme. ${ }^{8}$ Am 12. Februar 1946 verstarb Lorenz auf seinem Landsitz in Altenberg in Niederösterreich und wurde auf dem Ortsfriedhof in der Familiengruft beigesetzt.

${ }^{8}$ https://de.wikipedia.org/wiki/Adolf_Lorenz (Mediziner, 1854) 


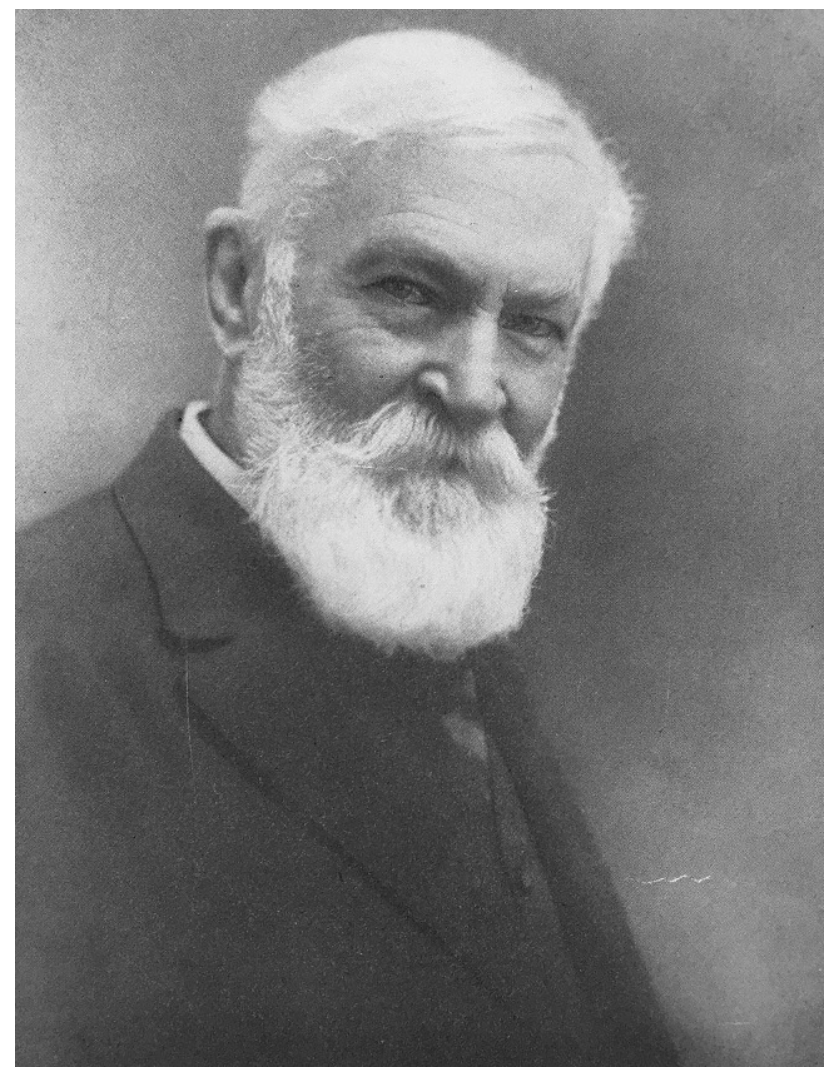

Abb. 9 Adolf Lorenz im höheren Alter. (Aus: [16])

\section{Schluss}

Damit endete eine medizinisch-historische Korrespondenzgeschichte zweier Persönlichkeiten, die in der Reichshauptstadt Wien begann und sich danach über zwei neue staatsrechtliche Regionen erstreckte, die sich viele Jahrhunderte in einem gemeinsamen Reich befunden hatten.

Danksagung Stellvertretend für die große Anzahl von Personen, die bei der Erstellung dieser Arbeit hilfreich und unterstützend waren, möchten sich die Autoren bei Wolfgang Riemer, Wien, Österreich, Anna Ohlidal, D. Wesel und Irena Kraitlová, alle Prag, Tschechien, herzlich bedanken. Die Autorin dankt auch dem Direktor des Masaryk-Institutes und des Archivs der Akademie der Wissenschaften der Tschechischen Republik Luboš Velek.

Förderung Die Arbeit entstand mit institutioneller Unterstützung RVO:67985921.

Funding Open access funding provided by Medical University of Vienna.

Interessenkonflikt G. Holzer und H. Kokešová geben an, dass kein Interessenkonflikt besteht.

Open Access Dieser Artikel wird unter der Creative Commons Namensnennung 4.0 International Lizenz veröffentlicht, welche die Nutzung, Vervielfältigung, Bearbeitung, Verbreitung und Wiedergabe in jeglichem Medium und Format erlaubt, sofern Sie den/die ursprünglichen Autor(en) und die Quelle ordnungsgemäß nennen, einen Link zur Creative Commons Lizenz beifügen und angeben, ob Änderungen vorgenommen wurden.

Die in diesem Artikel enthaltenen Bilder und sonstiges Drittmaterial unterliegen ebenfalls der genannten Creative Commons Lizenz, sofern sich aus der Abbildungslegende nichts anderes ergibt. Sofern das betreffende Material nicht unter der genannten Creative Commons Lizenz steht und die betreffende Handlung nicht nach gesetzlichen Vorschriften erlaubt ist, ist für die oben aufgeführten Weiterverwendungen des Materials die Einwilligung des jeweiligen Rechteinhabers einzuholen.

Weitere Details zur Lizenz entnehmen Sie bitte der Lizenzinformation auf http://creativecommons.org/licenses/by/4. $0 /$ deed.de.

\section{Literatur}

1. Shorsce CE. Fin-de-Siecle Vienna-Politics and Culture. NewYork: Alfred A. Knopf; 1980.

2. Masaryk TG. Der Selbstmord als sociale Massenerscheinung der modernen Civilisation. Wien: Konegen; 1881. https://archive.org/details/derselbstmordal00masagoog/ page/n5.

3. Holzer G. Adolf Lorenz' Entscheidung zum Medizinstudium. Wien MedWochenschr. 2017;167:126-30.

4. Lorenz A. Der schlechte Anatom. Eine märchenhafte, aber wahre Geschichte. Neue Freie Presse 4 Feb 1919;19557:1-3.

5. Doležal J. Masaryk - anatom [Masaryk - Der Anatom]. Tribuna 7 März 1920;58:4-6.

6. Lorenz A. Ueber eine unblutig-chirurgische Behandlung der angeborenen Hüftgelenks-Luxationen durch die Functionelle Belastungsmethode. Wien Klin Rundsch. 1896;10:76-7.

7. Lorenz A. Mein Schüler Masaryk. Neues Wiener Tagblatt 16 Sept 1937;256:7.

8. Lorenz A. Můj žák Masaryk [Mein Schüler Masaryk]. Lidové noviny 17 Sept 1937;469:1.

9. Lorenz A. Moje vzpomínky na dr. phil. T. G. Masaryka a na prvního presidenta Československé republiky [Meine Erinnerungen an Dr. phil. T. G. Masaryk und an den ersten Präsidenten der Tschechoslowakischen Republik]. Věstník československých lékařù. 1937;49:1719-20.

10. Lorenz A. Ich durfte helfen. Mein Leben und Wirken. Wien: Czernin; 2017.

11. Masaryk-Institut und Archiv der Akademie der Wissenschaften der Tschechischen Republik (Masarykův ústav a Archiv Akademie věd České republiky), Abteilung Archiv des Instituts von T. G. Masaryk (Archiv Ústavu T. G. Masaryka), Bestand T. G. Masaryk, Karton 700, Signatur KOR-II-7 und Karton 701, Signatur KOR-II-15.

12. Lorenz A. Špatný anatom. Pohádková, ale pravdivá historka [Der schlechte Anatom. Eine märchenhafte, aber wahre Geschichte]. Tribuna 6 Feb 1919;5:1-3.

13. Hlaváčková L, Masaryk TG a prof. MUDr. Adolf Lorenz [T. G. Masaryk und Prof. Dr. med. Adolf Lorenz]. Trendy v medicíně. 2000;2:98-9.

14. Kokešová H. Tomáš Garrigue Masaryk a ortoped Adolf Lorenz [Tomáš Garrigue Masaryk und Orthopäde Adolf Lorenz]. Práce z dějin Akademie věd. 2019;11:53-72. 
15. Tragl KH. Chronik der Wiener Krankenanstalten. Wien: Böhlau; 2007. S. 222. http://books.google.cz/books? id=u6PIdob9GzcC\&pg=PA222\&lpg=PA222\&dq=viktor+ mucha+1892+Wien\&source=bl\&ots=MD4tph2cPI\&sig= -VKEL2keYMaHwhz2J8196smWrok\&hl=cs\&sa=X\&ei= hztFU6uvD_Sw7Abx54GgBA\&ved=0CB4Q6AEwAA\#v= onepage\&q=viktor\%20mucha $\% 201892 \% 20$ Wien\&f=false.
16. Lorenz A. My life and work. New York: Charles Scribner's Sons; 1937.

Hinweis des Verlags Der Verlag bleibt in Hinblick auf geografische Zuordnungen und Gebietsbezeichnungen in veröffentlichten Karten und Institutsadressen neutral. 